\title{
Does cranberry have a role in catheter-associated urinary tract infections?
}

Dominique Thomas, $\mathrm{BS}^{1}$; Matthew Rutman, $\mathrm{MD}^{2}$; Kimberly Cooper, $\mathrm{MD}^{2}$; Andrew Abrams, $\mathrm{MPH}^{3}$; Julia Finkelstein, $\mathrm{MD}^{2}$; Bilal Chughtai, $\mathrm{MD}^{1}$

${ }^{1}$ Deptartment of Urology, Weill Cornell Medical College/New York Presbyterian Hospital, New York, NY, United States; ${ }^{2}$ Deptartment of Urology, Columbia University Medical Centre/New York Presbyterian Hospital, New York, NY, United States; ${ }^{3}$ Diana H. Jones Innovative Senior Center, Brooklyn NY, United States

Cite as: Can Urol Assoc J 2017; Epub ahead of print. http://dx.doi.org/10.5489/cuaj.4472

Published online November 1, 2017

$* * *$

\section{Abstract}

Introduction: Catheter-associated urinary tract infections (CA-UTIs) are a prevalent and costly condition, with very few therapeutic options. We sought to evaluate the efficacy of an oral cranberry supplement on catheter-associated urinary tracts infections over a six-month period. Methods: Subjects with long-term indwelling catheters and recurrent symptomatic CA-UTIs were enrolled to take a once-daily cranberry supplement - an oral cranberry supplement with 36 mg of the active ingredient proanthocyanidin (PACs). Primary outcome was reducing the number of symptomatic CA-UTIs. This was defined by $\geq 103$ (cfu)/mL of $\geq 1$ bacterial species in a single catheter urine specimen and signs and symptoms compatible with CA-UTI. Secondary outcomes included bacterial counts and resistance patterns to antibiotics.

Results: 34 patients were enrolled in the trial; 22 patients (mean age 77.22 years, $77.27 \%$ were men), completed the study. Cranberry was effective in reducing the number of symptomatic CAUTIs in all patients $(n=22)$. Resistance to antibiotics was reduced by $28 \%$. Furthermore, colony counts were reduced by $58.65 \%$. No subjects had adverse events while taking cranberry.

Conclusions: Cranberry reduced the number of symptomatic CA-UTIs, antibiotic resistances, and major causative organisms in this cohort. Larger, placebo-controlled studies are needed to further define the role of cranberry in CA-UTIs. 


\section{Introduction}

Catheter associated urinary tract infections (CA-UTIs) are a major public health issue. In the United States, these infections account for approximately $36 \%$ of health care related infections.[1] This leads to a large economic burden on the patient, provider, and healthcare system. Despite many proposed interventions to alleviate this, CA-UTIs rates have still remained high.[2] When patients are catheterized for more than seven days, 10-50\% will develop a CAUTI.[3]

Short of early removal of the catheter, there has been no further intervention to reduce rated of CA-UTIs. Thus, the search for an alternative treatment is of the utmost significance. Interestingly, the use of herbals have been utilized to treat urinary tract infections (UTIs) for centuries.[4] Cranberry, in its many forms, has been used for the prevention and treatment of UTIs.[5] The active components in cranberry, known as proanthocyanidins (PACs), are large condensed tannin molecules with A-type linkages exhibiting strong bacterial anti-adhesion activity.[6] These compounds prevent bacterial adhesion to the bladder wall, thereby preventing infection.[6] There are no studies on the efficacy of cranberry for those with CA-UTIs. We present the result of a proof of concept study evaluating the efficacy and safety of cranberry on CA-UTIs.

\section{Methods}

The study was conducted at 2 tertiary outpatient facilities and was approved by the Food and Drug Administration (FDA) and Institutional Review Board (IRB).

\section{Patient selection}

Subjects with indwelling catheters who had 2 documented symptomatic CA-UTIs over 6 months were included. For the purposes of this study, a symptomatic CA-UTI was defined by $\geq 10^{3}$ (cfu)/mL of $\geq 1$ bacterial species in a single catheter urine specimen[7] and signs and symptoms compatible with CA-UTI included new onset of fever, rigors, altered mental status, malaise, or lethargy with no other identified cause; hematuria; pelvic pain or dysuria.[7] If a patient developed a CA-UTI at any time during the study, they were treated using an appropriate course of antibiotics based on the sensitivity of their urine culture. Furthermore, the most prevalent isolated organisms were identified for each culture.

All subjects had to be at least 18 years of age. Subjects were not able to tolerate surgery or perform intermittent catheterization (Table 1). All catheters used in the study were standard latex catheters. No antiseptic catheters were used in this study. Subjects were excluded from the study previous allergic reaction to cranberry containing products, on Warfarin, on chronic antibiotics, performing daily bladder irrigation.

\section{Intervention}

Eligible subjects took one capsule of cranberry daily manufactured by Pharmatoka ${ }^{\circledR}$ for 6 consecutive months. This capsule contains $36 \mathrm{mg}$ of total cranberry extracted from juice (Vaccinium Macrocarpon Aiton) proanthocyanidins (PACs-A and B types together) as measured 
using the BL-DMAC method.[8] All subjects took the cranberry supplement at bedtime to avoid the alkaline tide of meals which could negatively affect the efficacy of cranberry. At each visit, urine culture, urinalysis, and symptoms of a CA-UTI were assessed. Catheters were changed at these monthly visits. Subjects were assessed to ensure there were no other exposures to antibiotics.

\section{Power calculation}

This is a pre/post study with the rate of UTI's in the prospectively-treated patients being compared to their rate of CA-UTI's in the 6 months prior to treatment (as determined by chart review). It recognized that this is a biased comparison due to the different UTI ascertainment methodologies in the two time periods (i.e., prospective identification vs. prior chart review). Therefore, the primary intent was to estimate the CA-UTI proportion in the prospectively-treated cohort. Since, $100 \%$ of patients had CA-UTIs to $80 \%$ with a prospective treatment to get a preliminary indication of efficacy. This study was designed to screen for an early indication of efficacy, rather than a definitive demonstration of cranberry. For this study, assuming an absolute reduction in the number of symptomatic CA-UTIs by $20 \%$ and with a type I error of 0.10 , a power of $0.80,95 \%$ of confidence intervals; the total sample size required to achieve these objectives is at least 20 subjects. With an attrition rate of 10\%, 22 subjects will be required.

\section{Statistical analysis}

Given this is a proof of concept study, all data collected was analyzed by descriptive statistics. Events and percentages were presented for patients' demographics and comorbidities. Mean and standard deviation were calculated for age. All analyses were performed in Stata Version 13.0 (StataCorp, College Station, TX).

\section{Results}

A total of 34 subjects were recruited for the study, and of this 22 subjects completed the study (See Figure 1 for reasons of attrition). The mean age was 77.22 years old (56-90 years old) (see Table 1). $77.27 \%(n=17)$ were males and $22.73 \%(n=22)$ were females. The Charlson AgeComorbidities Index (CACI) mean score was 4.909. See Table 1 for major comorbidities in this population. Table 2 shows the baseline characteristics for the subjects in the study.

At baseline the mean number of antibiotic resistance for the major causative organisms was $2.579 \pm 1.774$ (See Table 2). Figure 2 show the specific antibiotic resistances from baseline throughout the 6-months the patients were in the study (See Figure 2). At 6-months follow-up the mean number of antibiotic resistance for the major causative organisms had reduced to $1.857 \pm$ 1.215. This is an overall reduction by $28 \%$ while on the cranberry capsule.

All subjects at baseline had an average of $1.318 \pm 0.716$ major causative bacterial organisms in their urine cultures. At the end of the trial, this was reduced to $0.545 \pm 0.739$. This represents an overall reduction by $58.65 \%$ in bacterial organisms found in the study subjects' urine cultures (See Table 2). 
For the subjects in this study, at baseline their urinalysis showed a mean value of 51.944 \pm 41.886 WBC. At the end of the trial, subjects' urinalysis showed a mean WBC of $27.363 \pm$ 14.778 (See Table 2).

\section{Discussion}

This is the first study evaluating the use of cranberry in CA-UITs. While using this intervention during the 6-month trial, no subject presented with symptomatic CA-UTIs. The PACs present in cranberry are thought to inhibit P-fimbriated E. coli and other uropathogens from adhering to uroepithetial cells in the urinary tract.[9, 10]

Over 6-months, there was $28 \%$ reduction in all antibiotic resistance and a 58.65\% reduction in all major causative organisms. There was overall reduction in both the antibiotic resistance and the major causative organisms present in our subjects' urine cultures. This may be due to change in bacterial flora or the decrease use in antibiotics, which thereby reduced the resistant strains.

There have been very few other agents used to reduce CA-UTIs. Methenamine hippurate has been the most studied.[11] There have been a few studies on both bacteriuria and symptomatic CA-UTIs.[11] Methenamine hippurate had some benefit in preventing symptomatic CA-UTIs in patients without any renal tract abnormalities (RR 0.24, 95\% CI 0.07 to 0.89) as well as bacteriuria (RR 0.56, 95\% CI 0.37 to 0.83).[11] However, patients with a history of renal tract abnormalities were at a greater risk for symptomatic UTIs (RR 1.54, 95\% CI 0.38 to 6.20) and bacteriuria (RR 1.29, 95\% CI 0.54 to 3.07).[11] This treatment was only evaluated for a duration of a week, therefore it is difficult to extrapolate this data for 6-months. However, the overall effectiveness of this intervention remains unclear. The use of long-term antibiotics prophylactic has been used in patients with CA-UTIs.[12] Wazait et al. assessed the use of prophylactic antibiotics in patients with and without existing bacteriuria.[12] A total of 48 patients were enrolled. Within a 2-week time period, 3 patients were diagnosed with a CAUTIs.[12] During this time period, despite being on a 48-hour course of ciprofloxacin did not decrease the incidence of CA-UTIs.[12]

Almost all the data on CA-UTIs has been on novel catheter systems, such as silverimpregnated or antimicrobial catheters. However, the use of these catheters have not decreased the incidence of symptomatic CA-UTI rates.

This study was limited by a lack of a formal control group and thus did not compare the efficacy of this intervention juxtaposed to other treatment options. The investigators and the patients involved in this study were aware of the treatment allocation. This may have introduced bias as to which patients would be offered the cranberry. We had a sample size of 22 subjects completing the full study due to attrition and not adhering to the intervention guideline (i.e. not taking the cranberry supplement daily). This was likely due to this population being an older and sicker. Another limitation was using subjects as their own historic controls when comparing results.

Despite these limitations, our study is the first of its kind to use cranberry for prevention of a costly and common problem. Further, no study has long term 6-month data on preventing 
CA-UTIs. The effect of cranberry was durable during the study period. Further, cranberry was well tolerated with no unexpected adverse events or allergies.

\section{Conclusion}

In conclusion, after taking cranberry, no subjects presented with symptomatic CA-UTIs during the study period. Further, antibiotic resistances and patterns of major causative organisms decreased. This proof of concept study clearly demonstrates cranberry may be beneficial in this vulnerable and costly condition. There is an urgent need for larger placebo controlled trials. 


\section{References}

1. Rebmann T, Greene LR. Preventing catheter-associated urinary tract infections: An executive summary of the Association for Professionals in Infection Control and Epidemiology, Inc, Elimination Guide. American journal of infection control 2010; 38:644-6.

2. CDC. Healthcare-associated Infections (HAI) Progress Report | HAI | CDC. Available at: http://www.cdc.gov/hai/surveillance/progress-report/index.html.

3. Mody L, Greene MT, Saint S, et al. Comparing Catheter-Associated Urinary Tract Infection Prevention Programs Between Veterans Affairs Nursing Homes and NonVeterans Affairs Nursing Homes. Infect Control Hosp Epidemiol 2016:1-7.

4. Raz R, Chazan B, Dan M. Cranberry juice and urinary tract infection. Clinical infectious diseases : an official publication of the Infectious Diseases Society of America 2004; 38:1413-9.

5. Dieter AA. Cranberry capsules (2 taken twice daily for an average 38 days) reduce the risk of postoperative urinary tract infection in women undergoing benign gynaecological surgery involving intraoperative catheterisation. Evidence-based medicine 2015; 20:137.

6. Sanchez-Patan F, Bartolome B, Martin-Alvarez PJ, Anderson M, Howell A, Monagas M. Comprehensive assessment of the quality of commercial cranberry products. Phenolic characterization and in vitro bioactivity. Journal of agricultural and food chemistry 2012; 60:3396-408.

7. Hooton TM, Bradley SF, Cardenas DD, et al. Diagnosis, prevention, and treatment of catheter-associated urinary tract infection in adults: 2009 International Clinical Practice Guidelines from the Infectious Diseases Society of America. Clinical infectious diseases : an official publication of the Infectious Diseases Society of America 2010; 50:625-63.

8. Prior RL, Fan E, Ji H, et al. Multi-laboratory validation of a standard method for quantifying proanthocyanidins in cranberry powders. Journal of the science of food and agriculture 2010; 90:1473-8.

9. Tao Y, Pinzon-Arango PA, Howell AB, Camesano TA. Oral consumption of cranberry juice cocktail inhibits molecular-scale adhesion of clinical uropathogenic Escherichia coli. Journal of medicinal food 2011; 14:739-45.

10. Howell AB, Vorsa N, Der Marderosian A, Foo LY. Inhibition of the adherence of Pfimbriated Escherichia coli to uroepithelial-cell surfaces by proanthocyanidin extracts from cranberries. The New England journal of medicine 1998; 339:1085-6.

11. Lee BS, Bhuta T, Simpson JM, Craig JC. Methenamine hippurate for preventing urinary tract infections. The Cochrane database of systematic reviews 2012; 10:Cd003265.

12. Wazait HD, van der Meullen J, Patel HR, et al. Antibiotics on urethral catheter withdrawal: a hit and miss affair. The Journal of hospital infection 2004; 58:297-302. 


\section{Figures and Tables}

Fig. 1. Study enrollment and intervention allocation process. ${ }^{1}$ More than 1 criterion could apply to each individual. ${ }^{2}$ Participants received intervention of receiving a daily synthesized cranberry extract nutraceutical. Adhering to the protocol indicated taking the daily nutraceutical for 6 months and attending monthly physician visits for urine culture/urinalysis.

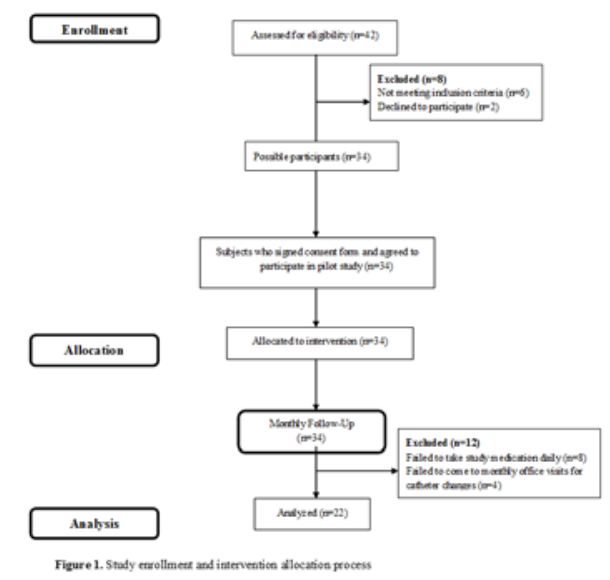

Fig. 2. Radar Scatterplot of the number of antibiotic resistances at baseline to 6-months while on a cranberry supplement. This figure represents the number of antibiotic resistances across the study period from month 0 until the end of the trial at month 6 . On the outside axis, the names of the specific antibiotics are labelled. A value in this case indicates a major causative organism was found in the patient's urine culture/urinalysis has resistance to the specific antibiotic. The yaxis represents the number of resistances. The larger the shaded area the more antibiotic resistances and the smaller the shaded area the less amount of antibiotic resistances.

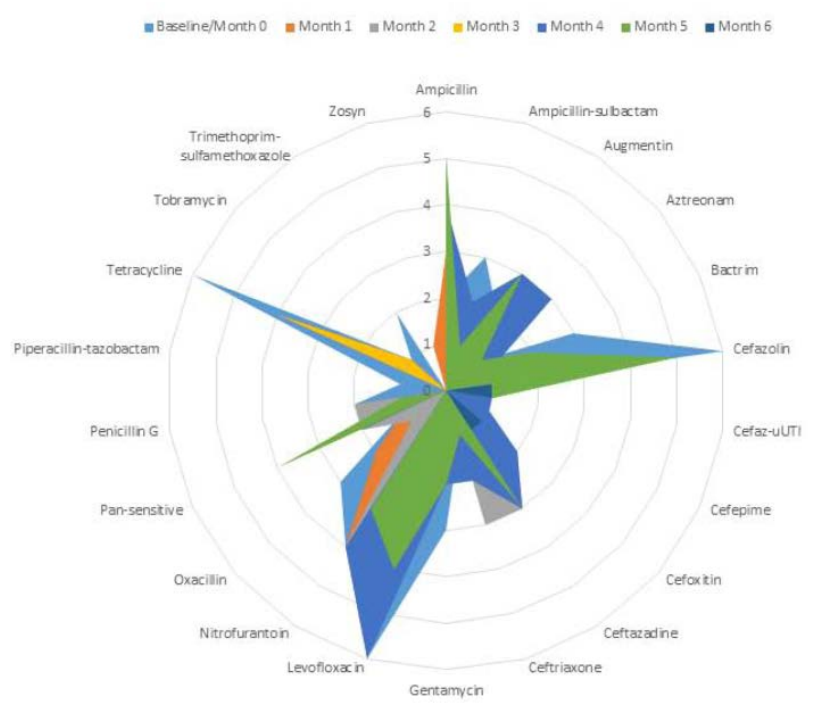




\begin{tabular}{|c|c|}
\hline Characteristic/demographic $^{1}$ & Mean results n $(\%)^{2}$ \\
\hline Age, mean \pm SD & $77.22 \pm 10.00$ yrs \\
\hline \multicolumn{2}{|l|}{ Sex } \\
\hline Male & $17(77.27 \%)$ \\
\hline Female & $5(22.72 \%)$ \\
\hline Common Comorbidities & Number of Patients (\%) \\
\hline Hypertension & $10(45.45 \%)$ \\
\hline Diabetes mellitus & $5(22.72 \%)$ \\
\hline Chronic Renal Failure & $3(13.64 \%)$ \\
\hline Congestive Health Failure & $5(22.72 \%)$ \\
\hline Stroke & $5(22.72 \%)$ \\
\hline Myocardial Infarction & $1(4.55 \%)$ \\
\hline Charlson Age-Comorbidities Index (CACI) & Mean results n $(\%)^{2}$ \\
\hline Mean & 4.909 \\
\hline Min & 2 \\
\hline Max & 10 \\
\hline Relative Risk of Death (RR) mean & 7.331 \\
\hline Reasons for Catheterization Males $(n=17)$ & Mean results $n(\%)^{2}$ \\
\hline Benign Prostatic Hyperplasia & $12(71 \%)$ \\
\hline Neurogenic Bladder & $5(29 \%)$ \\
\hline \multicolumn{2}{|l|}{ Reasons for Catheterization Females $(n=5)$} \\
\hline Detrusor Acontractility & $3(60 \%)$ \\
\hline 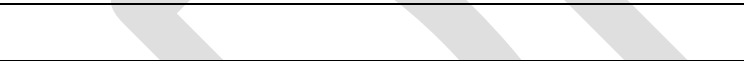 & 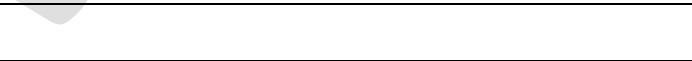 \\
\hline
\end{tabular}

${ }^{1}$ Characteristics/Demographics: The main patient characteristics we were interested in were reported, such as age, sex and CACI. ${ }^{2}$ All values are reported as a mean (average) unless stated otherwise. 


\begin{tabular}{|c|c|c|c|c|c|c|c|}
\hline $\begin{array}{l}\text { Urine Culture } \\
\text { /Urinalysis } \\
\text { Results }\end{array}$ & Baseline & Month 1 & Month 2 & Month 3 & Month 4 & Month 5 & Month 6 \\
\hline $\begin{array}{l}\text { Average } \\
\text { Number of } \\
\text { Antibiotic } \\
\text { Resistance }\end{array}$ & 2.579 & 2.4 & 2.625 & 2.181 & 2.667 & 2.625 & \begin{tabular}{|l|}
1.857 \\
\end{tabular} \\
\hline WBC U/A & 51.944 & 55.211 & 46.333 & 32.75 & 46.867 & 36.857 & 27.363 \\
\hline $\begin{array}{l}\text { Average } \\
\text { number of } \\
\text { Major } \\
\text { Causative } \\
\text { organisms }\end{array}$ & 1.318 & 1.091 & 1.181 & 0.910 & 1.000 & 1.045 & 0.545 \\
\hline Nitrite $^{2}$ & Negative & Negative & Negative & Negative & Negative & Negative & Negative \\
\hline
\end{tabular}

${ }^{1}$ Urine Culture/Urinalysis Results: We selected a few important components from our patients' urine cultures and urinalysis and reported them here. All values are reported as a mean (averages) unless stated otherwise. ${ }^{2}$ Nitrite: This value is reported as a binary negative or positive. 